\title{
New insights on pulsating white dwarfs from 3D radiation-hydrodynamical simulations
}

\author{
Pier-Emmanuel Tremblay ${ }^{1}$, Gilles Fontaine ${ }^{2}$, Hans-Günter Ludwig ${ }^{3}$, \\ Alexandros Gianninas ${ }^{4}$ and Mukremin Kilic ${ }^{4}$ \\ ${ }^{1}$ Department of Physics, University of Warwick, Gibbet Hill Rd., Coventry, CV4 7AL, UK \\ email: P-E.Tremblay@warwick.ac.uk \\ ${ }^{2}$ Département de Physique, Université de Montréal, C. P. 6128, Succursale Centre-Ville, \\ Montréal, QC H3C 3J7, Canada \\ ${ }^{3}$ Zentrum für Astronomie der Universität Heidelberg, Landessternwarte, Königstuhl 12, \\ D-69117 Heidelberg, Germany \\ ${ }^{4}$ Department of Physics and Astronomy, University of Oklahoma, 440 W. Brooks St., Norman, \\ OK, 73019, USA
}

\begin{abstract}
We have recently computed a grid of 3D radiation-hydrodynamical simulations for the atmosphere of pure-hydrogen DA white dwarfs in the range $5.0<\log g<9.0$. Our grid covers the full ZZ Ceti instability strip where pulsating DA white dwarfs are located. We have significantly improved the theoretical framework to study these objects by removing the free parameters of $1 \mathrm{D}$ convection, which were previously a major modeling hurdle. We present improved atmospheric parameter determinations based on spectroscopic fits with 3D model spectra, allowing for an updated definition of the empirical edges of the ZZ Ceti instability strip. Our 3D simulations also precisely predict the depth of the convection zones, narrowing down the internal layers where pulsation are being driven. We hope that these $3 \mathrm{D}$ effects will be included in asteroseismic models in the future to predict the region of the HR diagram where white dwarfs are expected to pulsate.
\end{abstract}

Keywords. White Dwarfs, Convection, Hydrodynamics, Asteroseismology

\section{Introduction}

The large majority of stars more massive than $1.5 M_{\odot}$ that have ever formed in the Milky Way are now slowly cooling as white dwarfs. These degenerate stars thus offer a wealth of information about the star formation history and initial mass function for intermediate mass stars that is inaccessible otherwise. Owing to their well constrained cooling rates, white dwarfs also provide a precise chronology of when the first stars in the galactic disk and halo have formed (see, e.g., Winget et al. 1987). However, the accuracy of the cooling age determinations is limited by our understanding of the layered interior of white dwarfs (Fontaine et al. 2001).

Asteroseismic analyses of white dwarfs offer a powerful tool to constrain the interior and cooling ages (see, e.g., Fontaine \& Brassard 2008, Althaus et al. 2010). It is especially the case since all hydrogen-atmosphere DA white dwarfs are expected to pulsate as ZZ Ceti stars once they cool and become convective owing to the large opacity of recombining hydrogen in the envelope. Until recently, spectroscopic and asteroseismic studies of ZZ Ceti white dwarfs were performed with theoretical tools employing the 1D mixinglength theory (MLT) of convection (Böhm-Vitense 1958). This phenomenological model of convection has little predictive power and different free parameters were employed for both applications. We present a novel approach using a more realistic treatment of convection with $3 \mathrm{D}$ radiation-hydrodynamics simulations. These simulations have much 


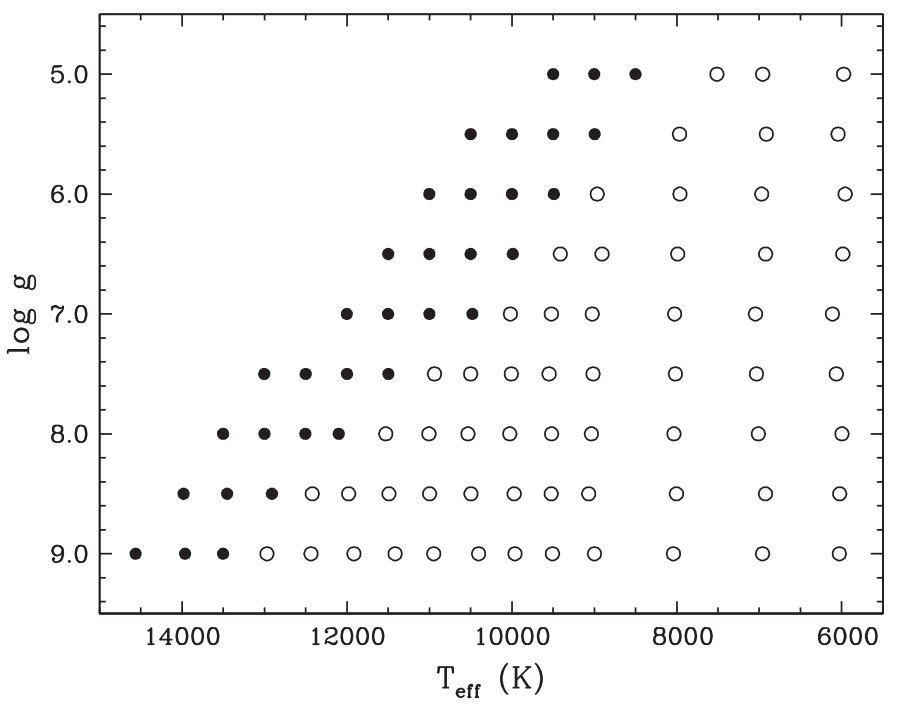

Figure 1. Surface gravity (logarithmic value in cgs units) and mean $T_{\text {eff }}$ for our $\mathrm{CO}^{5} \mathrm{BOLD} 3 \mathrm{D}$ model atmospheres. The simulations were computed with a bottom boundary layer that is open (open circles) or closed (filled circles) to convective flows.

more predictive power and we aim at verifying whether the current agreement between spectroscopic and asteroseismic studies has a solid physical foundation or is in part due to a particular choice of the 1D MLT parameters.

\section{Model Atmospheres}

We have carried out 94 simulations of pure-hydrogen WD atmospheres with $\mathrm{CO}^{5} \mathrm{BOLD}$ (Freytag et al. 2012). These 3D simulations are in the range $5.0 \leqslant \log g \leqslant 9.0$ and cover the vast majority of observed convective DA white dwarfs. The computations are further described in Tremblay et al. (2013) and references therein. Our calculations complement the CIFIST grid of $\mathrm{CO}^{5} \mathrm{BOLD}$ simulations for stars and giants (Caffau et al. 2011) with $1.0 \leqslant \log g \leqslant 4.5$.

Fig. 1 illustrates the 3D white dwarf simulations in a HR-type diagram. The warmest simulations, represented by filled symbols, have a convective zone that is thinner than the vertical dimension of the atmosphere. In those cases, we use a bottom layer that is closed to convective flows with imposed zero vertical velocities. In cooler models, the convective flux is transported in and out of the domain through an open boundary. In all cases the lateral boundaries are periodic and the top boundary is open to material flows and radiation. Further details on the numerics and boundary conditions are reported in Freytag et al. (2012). For the particular case of white dwarfs, we employ a grid of $150 \times 150 \times 150$ points, the Hummer \& Mihalas (1988) EOS, the Stark broadening profiles of Tremblay \& Bergeron (2009), and the quasi-molecular line opacity of Allard et al. (2004). The wavelength-dependent opacities are sorted in the 8 or 13 bin configurations discussed in Tremblay et al. $(2013,2015 b)$. 

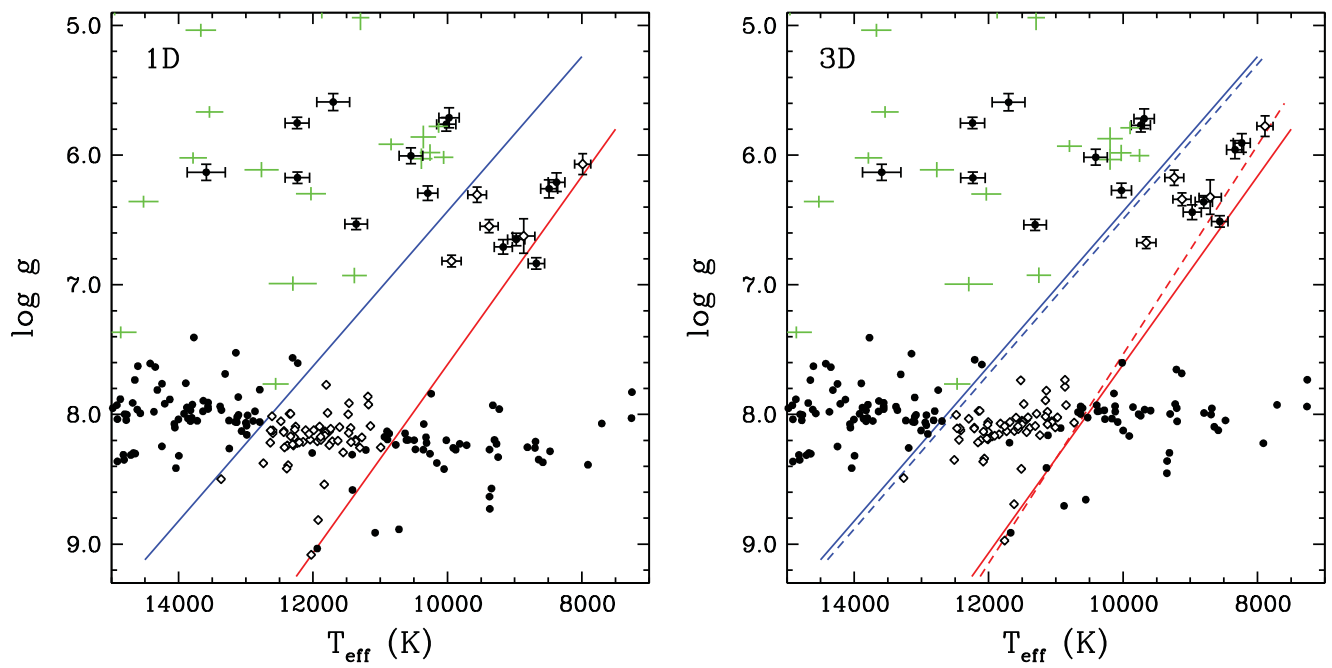

Figure 2. The ZZ Ceti instability strip based on the 1D (left panel, ML2/ $\alpha=0.8$ parameterization) and 3D (right panel) spectroscopic analysis. White diamonds represent the pulsators, whereas black dots show the photometrically constant WDs. The solid blue and red lines represent the empirical boundaries of the ZZ Ceti instability as determined by Gianninas et al. (2014). The dotted lines denote tentative boundaries matching the location of both instability strips (for normal mass and ELM pulsators) based on the 3D corrected parameters. The green error bars denote the remaining ELM WDs that have not yet been investigated for photometric variability.

\section{ZZ Ceti White Dwarfs}

\subsection{Spectroscopic Parameters}

We have computed mean 3D structures which are spatial and temporal averages of $T^{4}$ and $P$ performed over surfaces of constant Rosseland optical depth. These mean structures were employed to compute spectra, hereafter $\langle 3 \mathrm{D}\rangle$ spectra, using the same $1 \mathrm{D}$ radiative transfer code as that used for our latest grid of 1D spectra (Tremblay et al. 2011). We have verified that the numerical and physical approximations (i.e. the spatial and temporal averages, the grid resolution, and the opacity binning) leading to the $\langle 3 \mathrm{D}\rangle$ spectra do not change the derived atmospheric parameters by more than $1 \%$, effectively removing the free parameters previously inherent to the MLT. One notable exception is the extremelylow-mass $($ ELM) regime $(\log g \lesssim 6.5)$ where the derived 3D atmospheric parameters are uncertain by up to $5 \%$ (Tremblay et al. 2015b). In the following, we employ the convenient $1 \mathrm{D}$ to $3 \mathrm{D} T_{\text {eff }}$ and $\log g$ correction functions presented in Tremblay et al. $(2013,2015 \mathrm{~b})$ to compare the 1D and 3D parameters of ZZ Ceti white dwarfs.

Fig. 2 shows the instability strip for the pulsating DA white dwarfs from a uniform analysis by Gianninas et al. $(2011,2014)$ with high signal-to-noise observations. We present the results for both 1D and 3D atmospheric parameters. We also show the five ELM pulsators currently known, and a large number of photometrically constant stars that help define the boundaries of the instability strip. The solid lines show the boundaries of the instability strip from Gianninas et al. (2014).

There is a clear trend of smaller $\log g$ values when the $3 \mathrm{D}$ corrections are applied, resulting in $\Delta \log g=-0.1$ for pulsating ZZ Ceti white dwarfs when compared to 1D models. The 3D corrections suggest an average $\log g$ value of $\sim 8.0$ for both pulsating and non-pulsating white dwarfs, confirming that we observe different evolutionary stages of 
the same population. On the other hand, the 1D models predict spurious high gravities for pulsating white dwarfs.

The blue edge of the instability strip is well defined in the 1D analysis. The 3D corrections move the stars systematically to slightly cooler temperatures and lower surface gravities. The dashed lines show the revised boundaries based on the 3D corrected parameters. For the blue edge, the boundary is simply shifted to the right by a small value of $100 \mathrm{~K}$. The blue edge of the instability strip is still well defined for both normal mass and ELM WDs in the 3D analysis. The red edge now has a higher slope, although given the small number of ELM pulsators currently known, it is too early to definitively constrain the red edge of the instability strip for those stars. The equations of the revised blue and red edges are defined by Equations 3.1 and 3.2, respectively.

$$
\begin{aligned}
& (\log g)_{\text {blue }}=5.96923 \times 10^{-4}\left(T_{\text {eff }}\right)_{\text {blue }}+0.52431 \\
& (\log g)_{\text {red }}=8.06630 \times 10^{-4}\left(T_{\text {eff }}\right)_{\text {red }}-0.53039
\end{aligned}
$$

\subsection{Asteroseismic Parameters}

Non-adiabatic asteroseismic models provide predictions for the position of the blue edge of the ZZ Ceti instability strip, although the results are highly sensitive to the parameterization of convection (Fontaine et al. 1994, Gautschy et al. 1996). Recently, van Grootel et al. (2012) relied on a non-adiabatic code including time-dependent convection to study the driving mechanism. Compared to earlier studies, their approach neither assumes frozen convection nor an instantaneous convection response during a pulsation cycle. Using 1D white dwarf structures with the ML2/ $\alpha=1.0$ parameterization of the MLT (Bohm \& Cassinelli 1971), they find a seismic blue edge at $T_{\text {eff }}=11,970 \mathrm{~K}$ for $\log g=8$, in fairly good agreement with the empirical blue edge from $1 \mathrm{D}$ and $3 \mathrm{D}$ spectroscopic analyses.

To reach a better internal consistency between the spectroscopic and asteroseismic results, it is desirable to replace the constant MLT parameterization employed in the asteroseismic models by a proper calibration from realistic 3D simulations. In order to perform this task, we have extracted the hydrogen mass included in the convection zones from our 3D simulations (Tremblay et al. 2015a). We note, however, that while the MLT only provides a bottom boundary of the convection zone based on the Schwarzschild criterion, the 3D stratifications are more complex with lower overshoot layers. Since the convective flux contribution at the bottom of the convection zone is critical in the nonadiabatic perturbation equations, here we define the bottom of the convection zone as the layer where the convective flux is zero (Tremblay et al. 2015a). Fig. 3 shows the 1D MLT envelope that best reproduces the $3 \mathrm{D}$ results as a function of $T_{\text {eff }}$ and $\log g$. For $T_{\text {eff }}$ values close to and above the blue edge, the $1 \mathrm{D}$ MLT is unable to reproduce the inneficient (small convective flux) but geometrically extended 3D convection zones with strong lower overshoot. As a consequence, the rapid change of the 1D MLT parameterization as a function of $T_{\text {eff }}$ should be taken with caution.

Fig. 4 shows the 1D MLT parameterization that best reproduces the 3D size of the convection zone along the empirical blue and red edges of the ZZ Ceti instability strip from Fig. 2. We find that the optimal ML2/ $\alpha$ value is never far from unity, in close agreement with the value generally used in asteroseismic applications. This confirms that the current agreement between the empirical and asteroseismic blue edges has a solid physical foundation and is not forced by specific choices of the 1D MLT parameters. Nevertheless, a proper account of the 3D effects as observed in Fig. 4 could further improve the agreement between asteroseismic and spectroscopic results. 


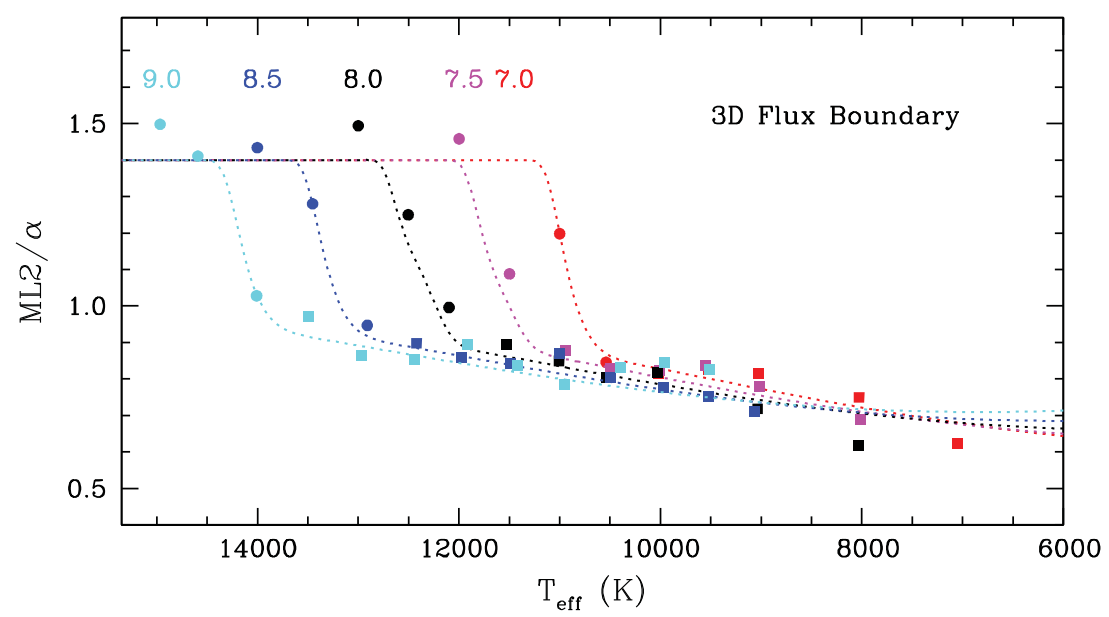

Figure 3. Calibration of ML2/ $\alpha$ for the lower part of the convection zone as a function of $T_{\text {eff }}$ and $\log g$ (represented by different colors with the legend at the top). The calibration is based on the 1D model that best replicates the zero point of convective flux at the bottom of a 3D simulation, either from a direct comparison (open circles) or by using the asymptotic entropy value of the deep inflowing material (filled squares, see Tremblay et al. 2015a for details). The dotted lines correspond to the fitting function proposed in Tremblay et al. (2015a).

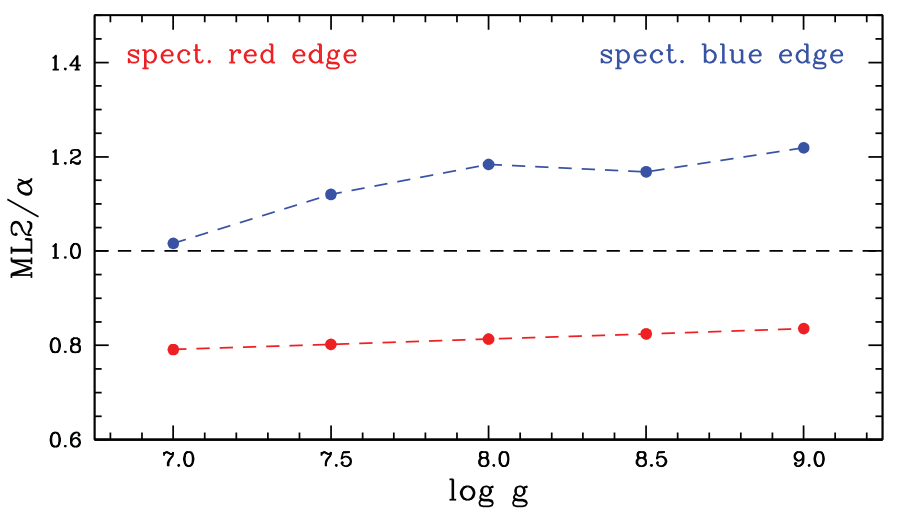

Figure 4. Similar to Fig. 3 but for the $T_{\text {eff }}$ values that correspond to the empirical blue and red edges of the ZZ Ceti instability strip, respectively (Eq. 3.1 and 3.2), as a function of $\log g$. The ML2/ $\alpha$ calibration is based on the 1D model that best replicates the zero point of convective flux at the bottom of a 3D simulation. The points are connected for clarity. The black dashed line represents the ML2 $/ \alpha=1.0$ value generally employed in asteroseismic applications.

\section{Conclusions}

We have presented a study of pulsating ZZ Ceti white dwarfs relying on improved 3D radiation-hydrodynamics simulations of the atmospheres. For pulsating white dwarfs, the convection zone is shallow enough that in many cases it is possible to model the full vertical extent of the convection zone within the $3 \mathrm{D}$ simulation, including the bottom layers which are important for pulsation driving. This allows for a much more consistent theoretical framework where surface and envelope convection are not treated with a different 1D MLT parameterization as it is usually done. We find that 3D spectroscopic parameters of ZZ Ceti stars are significantly different with an average shift of -0.1 dex in $\log g$ compared to $1 \mathrm{D}$, although the blue and red edges of the instability strip are very similar to the latest 1D determinations. 
We find that asteroseismic models would need to change the 1D MLT convective efficiency by $+20 \%$ and $-20 \%$ to match the $3 \mathrm{D}$ size of the convection zone near the blue and red edges of the instability strip, respectively. Accounting for these $3 \mathrm{D}$ effects could improve the agreement between the empirical (spectroscopic) and asteroseismic blue edges. Nevertheless, 3D effects are relatively mild, and the ML2 $/ \alpha=1.0$ parameterization currently employed in asteroseismic models is an appropriate characteristic value for the ZZ Ceti instability strip. It would be interesting to review the non-adiabatic pulsation calculations with the new calibrated $1 \mathrm{D}$ envelopes or a direct use of the $3 \mathrm{D}$ convective flux profiles (Gautschy et al. 1996). Finally, dynamical convection effects that are missing from both current and newly calibrated 1D envelopes could also have an impact on pulsations (van Grootel et al. 2012).

\section{References}

Allard, N. F., Kielkopf, J. F., \& Loeillet, B. 2004, A\&A, 424, 347

Althaus, L. G., Córsico, A. H., Isern, J., \& García-Berro, E. 2010, A\&̊AR, 18, 471

Böhm-Vitense, E. 1958, ZAp, 46, 108

Böhm, K. H. \& Cassinelli, J. 1971, A\& A, 12, 21

Caffau, E., Ludwig, H.-G., Steffen, M., Freytag, B., \& Bonifacio, P. 2011, Sol. Phys., 268, 255

Fontaine, G., Brassard, P., Wesemael, F., \& Tassoul, M. 1994, ApJ (Letters), 428, L61

Fontaine, G., Brassard, P., \& Bergeron, P. 2001, PASP, 113, 409

Fontaine, G. \& Brassard, P. 2008, PASP, 120, 1043

Freytag, B., Steffen, M., Ludwig, H.-G., et al. 2012, Journal of Computational Physics, 231, 919

Gautschy, A., Ludwig, H.-G., \& Freytag, B. 1996, A\& A, 311, 493

Gianninas, A., Bergeron, P., \& Ruiz, M. T. 2011, ApJ, 743, 138

Gianninas, A., Dufour, P., Kilic, M., et al. 2014, ApJ, 794, 35

van Grootel, V., Dupret, M.-A., Fontaine, G., et al. 2012, A\&̋A, 539, A87

Hummer, D. G. \& Mihalas, D. 1988, ApJ, 331, 794

Tremblay, P.-E. \& Bergeron, P. 2009, ApJ, 696, 1755

Tremblay, P.-E., Bergeron, P., \& Gianninas, A. 2011, ApJ, 730, 128

Tremblay, P.-E., Ludwig, H.-G., Steffen, M., \& Freytag, B. 2013, A\&\&A, 559, A104

Tremblay, P.-E., Ludwig, H.-G., Freytag, B., et al. 2015a, ApJ, 799, 142

Tremblay, P.-E., Gianninas, A., Kilic, M., et al. 2015b, ApJ, 809, 148

Winget, D. E., Hansen, C. J., Liebert, J., et al. 1987, ApJ (Letters), 315, L77 